\title{
MiRNA-125a-3p is a negative regulator of the RhoA-actomyosin pathway in A549 cells
}

\author{
BO HUANG ${ }^{1,2}$, WENTING LUO ${ }^{1}$, LIMEI SUN ${ }^{1}$, QINGFU ZHANG ${ }^{1}$, LILI JIANG ${ }^{1,3}$, \\ JIHONG CHANG $^{1}$, XUESHAN QIU ${ }^{1}$ and ENHUA WANG ${ }^{1}$
}

\begin{abstract}
${ }^{1}$ Department of Pathology, First Affiliated Hospital and College of Basic Medical Sciences, China Medical University, Heping, Shenyang, Liaoning 110001; ${ }^{2}$ Department of Pathology, Liaoning Tumor Hospital, Dadong, Shenyang,

Liaoning 110042; ${ }^{3}$ Department of Pathology, Medical College of Eastern Liaoning University, Dandong, Liaoning 118000, P.R. China
\end{abstract}

Received December 16, 2012; Accepted February 15, 2013

DOI: $10.3892 /$ ijo.2013.1861

\begin{abstract}
MicroRNAs (miRNAs) function as genetic modulators that regulate gene expression, and are, thus, involved in a wide range of biological roles, including tumor cell migration and invasion. MiR-125a-3p is a mature form of miR-125a, derived from the 3'-end of pre-miR-125a. Our group has previously reported that $\mathrm{miR}-125 \mathrm{a}-3 \mathrm{p}$ functions as a tumor suppressor gene that inhibits the migration and invasion of lung cancer cells. Here, we report the discovery of a new regulatory layer of the RhoA-actomyosin pathway through which miR-125a-3p controls tumor cell migration. Overexpression of miR-125a-3p by transfection of sense-miR-125a-3p resulted in decreased RhoA protein levels, while the levels of RhoA mRNA remained constant. The concentrations of both RhoA-GTP protein and actin filaments decreased after miR-125a-3p overexpression in the A549 lung cancer cell line. Conversely, knockdown of miR-125a-3p by transfection of antisense-miR$125 \mathrm{a}-3 \mathrm{p}$ resulted in increased RhoA protein levels while the levels of RhoA mRNA remained unchanged. However, the concentration of both RhoA-GTP protein and actin filaments increased. To further demonstrate that RhoA is a potential target of miR-125a-3p, luciferase reporter constructs containing the RhoA 3'UTR demonstrated reduced reporter activity after ectopic expression of miR-125a-3p. Moreover, luciferase reporter constructs containing the RhoA 3'UTR mutant did not show significantly changed reporter activity. Furthermore, A549 cells demonstrated reduced migratory capacity after treatment with the Rho inhibitor CT04. Our results indicate that the loss
\end{abstract}

Correspondence to: Professor Xueshan Qiu, Department of Pathology, First Affiliated Hospital and College of Basic Medical Sciences, China Medical University, No. 92 North Second Road, Heping District, Shenyang, Liaoning 110001, P.R. China E-mail: qiuxues@hotmail.com

Key words: lung cancer, migration, microRNA, miR-125a-3p, RhoA, RhoA-GTP of miR-125a-3p-controlled regulation of the RhoA-actomyosin pathway can lead to increased migration of tumor cells because of the upregulation of RhoA expression. In particular, an increased intracellular concentration of RhoA-GTP protein in A549 cells leads to the accumulation of actin filaments. These results provide new insights into the role of the miR-125a family in lung cancer.

\section{Introduction}

Most patients with malignant tumors do not die of the primary neoplasia, but of secondary tumors known as metastases. As early as 1996, Sporn reported that more than $90 \%$ of cancer deaths resulted from the development of metastatic lesions (1). Metastasis depends upon the processes of tumor cell migration and invasion. Today, the molecular and cellular fundamentals of migration and invasion are recognized as complex multistep biological processes, most probably regulated by distinct genes and signaling pathways at every step. The various cytoplasmic proteins and transcription factors mediating these processes have already been identified (2). It was recently discovered that miRNAs can affect a wide range of biological functions, including tumor cell invasion and metastasis (3-7). MiRNAs are small, endogenous, non-protein-coding single-stranded RNAs of 20-25 nucleotides that post-transcriptionally regulate the expression of hundreds of target genes. MiRNAs bind target mRNA sequences through canonical base pairing between the seed sequence, which includes nucleotides 2-8 from the 5 '-end, and the complementary sequence found in the 3 'UTR of its target mRNA (8). In mammals, mature miRNAs are integrated into an RNA-induced silencing complex, which can suppress translation and occasionally also induce degradation of a targeted mRNA. MiRNAs are primarily negative gene regulators of post-transcriptional repression, and targets include tumor suppressors and oncogenes. For example, miR-21 was found to be highly expressed in colorectal cancers, and induced metastasis and invasion by inhibiting the tumor suppressor gene PDCD4 in colorectal cancer cells (9). Thus, as an oncogene, miR-21 exhibited regulatory effects. However, when expressed at lower levels in tumors, miRNAs have also demonstrated 
tumor suppressor functions. For example, the action of let-7 miRNA as an antioncogene was found to be absent in lung cancers by inhibiting downstream translation of MYC (10).

MiR-125a has been reported to control the differentiation of the embryonic cancer cell line P19 by negatively regulating the 3'UTR of lin-28 mRNA (11). It was found to inhibit translation of the target gene t-trkC and thereby modulating proliferation of neuroblastomas (12). In addition, during the process of epithelial-mesenchymal transdifferentiation (EMT) in ovarian cancer, miR-125a was found to negatively regulate the target gene ARID3B and inhibit EMT (13).

MiR-125a-3p, a member of the miR-125a family, is derived from the 3'-end of pre-miR-125a. However, there are few reports on the function of miR-125a-3p. Our research group has shown that miR-125a-3p is poorly expressed in non-small cell lung cancers (NSCLCs) (14). Real-time polymerase chain reaction (PCR) assays showed that miR-125a-3p expression was significantly decreased in NSCLCs compared to corresponding adjacent normal tissue. Therefore, we hypothesized that miR-125a-3p may act as a tumor suppressor by negatively regulating some oncogenes. Importantly, Spearman's correlation tests demonstrated a negative relationship between miR-125a-3p expression, pathological tumor stage and lymph node metastasis. In addition, downregulation of levels of miR-125a-3p expression in A549 cells resulted in higher rates of invasion and migration. These previous results led us to hypothesize that miR-125a-3p may be involved in the migration and invasion of NSCLC tumor cells. To the best of our knowledge, there have not been any reports on the mechanisms involved in the regulation of migration and invasion by miR-125a-3p.

The metastatic sequence of primary tumor cells includes detachment, local migration and invasion of stromal tissue, intravasation and transit through blood vessels, capillary bed arrest and extravasation, further local crawling and invasion, attachment, formation of micrometastases, survival, perhaps dormancy and eventually additional proliferation $(15,16)$. Cellular migration is an essential function of tumor cell invasion and metastasis. Accordingly, the purpose of this study was to explore how miR-125a-3p affects the migration of cells of the A549 lung cancer cell line.

\section{Materials and methods}

Cell culture. A549 cells were propagated in DMEM (Gibco, Carlsbad, CA, USA) supplemented with $10 \%$ fetal bovine serum (FBS), $100 \mathrm{U} / \mathrm{ml}$ penicillin and $100 \mathrm{U} / \mathrm{ml}$ streptomycin. The cells were incubated at $37^{\circ} \mathrm{C}$ in a $5 \% \mathrm{CO}_{2}$ humidified atmosphere until $75 \%$ confluent.

Transfection. All transfections were carried out using Lipofectamine 2000 (Invitrogen, Carlsbad, CA, USA) according to the manufacturer's instructions. The following 2'-O-methyl oligonucleotides were synthesized by Integrated DNA Technologies and purchased from Shanghai GeneChem: 2'-O-Me-sense-125a-3p: 5'-ACA GGU GAG GUU CUU GGG AGCC-3', 2'-O-Me-antisense-125a-3p: 5'-GGC UCC CAA GAA CCU CAC CUGU-3', and 2'-O-Me-scramble-125a-3p: 5'-GGU CGG UGC UCG AUG CAG GUAA-3'. Twenty-four hours prior to transfection, the cells were plated into 6-well plates for RNA extraction and into $10-\mathrm{cm}$ dishes for protein extraction.
A549 cells were transfected with $20 \mu \mathrm{M}$ of 2'-O-methyl oligonucleotides, and RNA and protein were extracted $24 \mathrm{~h}$ after transfection. In addition, RhoA small interfering RNA (siRNA) or the negative control was used in co-transfection with or without antisense-125a-3p. The sequences of the RhoA siRNAs were: 5'-GAAGUCAAGCAUUUCUGUCTT-3' (sense) and 5'-GACAGAAAUGCUUGACUUCTT-3' (antisense) (17).

To block the function of RhoA, the Rho inhibitor CT04 (Cytoskeleton, Denver, CO, USA), was added to the culture medium at a final concentration of $2.0 \mu \mathrm{g} / \mathrm{ml}$, as described previously (18). Migration was investigated in the presence of CT04 using a Transwell chamber as described in the following section.

Cell migration assay. A549 cells ( $5 \times 10^{4}$ cells/chamber) were trypsinized, washed, resuspended in serum-free DMEM, and placed in the top portion of the chamber. The lower portion of the chamber contained $10 \% \mathrm{FBS}$ as a chemoattractant. The chambers were incubated at $37^{\circ} \mathrm{C}, 5 \% \mathrm{CO}_{2}$ for $12 \mathrm{~h}$, and then the cells remaining on the membrane were washed with PBS, fixed in $100 \%$ methanol, stained with haematoxylin, photographed and counted. Five random fields were selected randomly, then analyzed for each chamber. Each of 3 independent experiments was carried out in duplicate.

Luciferase assays. The plasmid used for these assays contained a full-length RhoA 3'UTR coupled to a luciferase reporter, and was a kind gift from Dr William Kong and Dr Jin Q. Cheng (H. Lee Moffitt Cancer Center and Research Institute, Tampa, FL, USA). The following primers were used to amplify the 3'UTR of the RhoA gene from human cDNA (NM_001664.2): forward primer 5'-GAC TAG TCA ATC TGG GTG CCT TGT CTTG-3', reverse primer 5'-CCC AAG CTT GGG TGC CTT TAT TCT ATT AGT AGT TGG AAA-3'. The PCR fragment were digested and cloned into the pMIR-Report vector (Ambion) at the SpeI and HindIII sites. The mutant plasmid containing a full-length RhoA 3'UTR mutant coupled to a luciferase reporter was constructed by Sauer Biotechnology Inc. (China).

A549 cells were seeded onto 24-well plates the day prior to transfection. They were transfected with $0.4 \mu \mathrm{g}$ of the luciferase expression construct, $20 \mu \mathrm{M}$ 2'-O-methyl sense or scramble oligonucleotides, and $0.02 \mu \mathrm{g}$ of the Renilla luciferase vector pRL-TK (Promega) for normalization. Mock transfected cells were transfected with the luciferase constructs alone. After $24 \mathrm{~h}$, luciferase assays were performed using the Dual-Luciferase Reporter Assay System (Promega). Firefly luciferase activity was normalized to Renilla luciferase activity. All transfection experiments were conducted in triplicate.

RNA isolation and quantitative real-time polymerase chain reaction ( $q R T-P C R)$. Total RNA was prepared using TRIzol (Invitrogen). RhoA cDNA was generated by PrimerScript RT reagent kit (Takara, Dalian, China) and amplified using RhoA primers with SYBR Premix EX Taq II (Takara). The primer sequences for RhoA were: 5'-CGA CAG CCC TGA TAG TTT A-3' (forward) and 5'-GTG CTC ATC ATT CCG AAG A-3' (reverse), and the primer sequences for $\beta$-actin were F: 5'-AGCACAGAGCCTCGCCTTTG-3' (forward), R: 5'-ACATGCCGGAGCCGTTGT-3' (reverse). The relative quantity of RhoA, normalized to $\beta$-actin, calculated based on the following equation $\mathrm{RQ}=2^{-\Delta \Delta \mathrm{Ct}}$. 
Western blot analysis. Cells were washed twice with ice-cold phosphate-buffered saline (PBS) and lysed using the M-PER reagent (Pierce Biotechnology) containing $1 \mathrm{mM}$ PMSF and phosphatase inhibitors for $1 \mathrm{~h}$ at $4^{\circ} \mathrm{C}$. The supernatants were centrifuged at $12,000 \mathrm{xg}$ for $30 \mathrm{~min}$ at $4^{\circ} \mathrm{C}$, and collected for analysis. Aliquots of supernatant containing $80 \mu \mathrm{g}$ of total protein were separated on 12 or $10 \%$ SDS-PAGE gels and transferred to PVDF membranes. The membranes were blocked using $5 \%$ fat-free milk, and incubated with mouse anti-RhoA (1:200, Santa Cruz Biotechnology, Santa Cruz, CA, USA), or mouse anti-GAPDH $(1: 1,000$, Santa Cruz Biotechnology). Membranes were incubated at $4^{\circ} \mathrm{C}$ overnight and incubated with corresponding secondary antibodies (1:4,000, Chemicon, Temecula, CA, USA) at room temperature for $1 \mathrm{~h}$. Immunoreactive bands were identified using Super ECL reagent (Pierce Biotechnology), according to the manufacturer's protocol. Specific bands for RhoA, F-actin and GAPDH were identified using prestained protein molecular weight markers (SM0441, MBI Fermentas). The ECL Imaging System (UVP Inc.) was used to visualize the specific bands and the optical density of each band was measured using Image J software.

Wound healing assay. When cell confluence was less than $90 \%$ at $48 \mathrm{~h}$ after transfection, wounds were created in confluent cells using a $200-\mathrm{ml}$ pipette tip. The cells were then rinsed with medium to remove any free-floating cells and debris. Medium was then added, and culture plates were incubated at $37^{\circ} \mathrm{C}$. Wound healing within the scrape line was observed at different time points, and representative scrape lines for each cell line were photographed. Duplicate wells for each condition were examined for each experiment, and each experiment was repeated 3 times. The optical the distance of wound were measured using Image $\mathbf{J}$ software.

Rho-GTP pull-down assay. The Rho-GTP pull-down assay was performed using an RhoA/Rac1/Cdc42 Activation Assay Combo Kit (STA-405, Cell Biolabs Inc., San Diego, CA, USA). Medium was aspirated off cells cultured to approximately 80 to $90 \%$ confluence, and the cells were rinsed twice with ice-cold PBS before being treated with ice-cold lysis buffer $(0.5-1 \mathrm{ml}$ per $100-\mathrm{mm}$ tissue culture plate). The cell culture plates were subsequently placed on ice for 10-20 min. The cells were centrifugated at $14,000 \mathrm{xg}$ for $10 \mathrm{~min}$ at $4^{\circ} \mathrm{C}$, and then $20 \mu \mathrm{l}$ of $0.5 \mathrm{M}$ EDTA was added to each aliquot sample. The positive and negative controls received $10 \mu \mathrm{l}$ of 100X GTP $\gamma$ S and $10 \mu 1$ of 100X GDP, respectively. All tubes were incubated with agitation for $30 \mathrm{~min}$ at $30^{\circ} \mathrm{C}$. Reactions were stopped using $65 \mu 1$ of $1.0 \mathrm{M} \mathrm{MgCl}_{2}$. Rhotekin RBD or PAK PBD Agarose beads were added to the cell lysates and incubated for $1 \mathrm{~h}$ at $4^{\circ} \mathrm{C}$. After centrifugation for $10 \mathrm{sec}$ at $14,000 \mathrm{x} \mathrm{g}$, the beads were washed with lysis buffer and Rho-GTP was eluted in Laemmli sample buffer. Antibody against RhoA was used to analyze eluted sample on western blots.

Immunofluorescence. When cell confluence was less than $5 \times 10^{5}$ cells at $48 \mathrm{~h}$ after transfection, cells were serum-starved for $4 \mathrm{~h}$ and fixed in $2 \%$ formaldehyde/PBS for $7 \mathrm{~min}$ at room temperature. After permeabilization with $0.1 \%$ Triton X-100 and

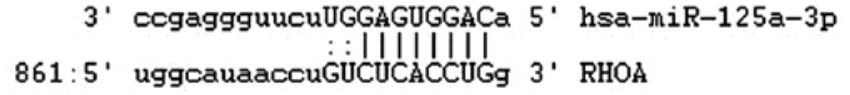

Figure 1. Predicted and confirmed miR-125a-3p target. RhoA 3'UTR contains conserved regions that may serve as a binding site for miR-125a-3p as determined by the miRanda algorithm.

blocking with $1.5 \%$ normal goat serum, the cells were stained with rhodamine-phalloidin for $30 \mathrm{~min}$ at $37^{\circ} \mathrm{C}$ to label F-actin, and mounted using Gelvatol mounting medium.

Statistical analysis. All data are presented as mean \pm SD of 3 independent experiments. A p-value $\leq 0.05$ was considered significant. All statistical analyses were performed using the SPSS13.0 software package.

\section{Results}

RhoA is a potential miR-125a-3p target gene. To study how miR-125a-3p might regulate migration, we first proceeded to identify potential targets known to play a role in cell mobility. Among the candidates surveyed previously (14), we found that the 3'UTR of the RhoA gene contains highly conserved regions that may serve as binding sites for miR-125a-3p, as determined at microrna.org (Fig. 1).

Effects of miR-125a-3p on RhoA expression. Since results of our previous study showed that miR-125a-3p expressed moderately in A549 NSCLC cells, we chose A549 cells for this study. To confirm whether suppression of migration by miR-125a-3p is associated with changes in RhoA expression, we measured the level of both RhoA mRNA and protein using qRT-PCR and western blot analysis in the presence of upregulation and downregulation of miR-125a-3p. Upregulation of miR-125a-3p was achieved using transfected sense-miR-125a-3p; while RhoA mRNA remained unchanged $(\mathrm{p}=0.309$, Fig. $2 \mathrm{~A})$, the RhoA protein concentration decreased (p<0.001, Fig. 2B). Downregulation of miR-125a-3p was achieved using transfected antisense-miR-125a-3; while RhoA mRNA remained unchanged ( $p=0.942$, Fig. $2 C$ ), the RhoA protein concentration increased $(\mathrm{p}<0.001$, Fig. 2D). These results indicate that miR-125a-3p may post-transcriptionally regulate RhoA expression.

MiR-125a-3p directly regulates the expression of the target gene RhoA. To further demonstrate that RhoA is a potential target of miR-125a-3p, we generated luciferase reporters that contained the 3'UTR of the RhoA gene (Fig. 3A). Results from 3 independent experiments showed that reporter activity was reduced by the ectopic expression of miR-125a-3p ( $<<0.001$, Fig. 3B). We also generated luciferase reporters that contained a mutated sequence within the predicted target sites of the 3'UTR of the RhoA gene (Fig. 3A) to further demonstrate the interaction between miR-125a-3p and the 3'UTR of RhoA. Data showed that reporter activity was not reduced by the ectopic expression of miR-125a-3p (Fig. 3B). Taken together, these results indicated that in A549 cells, the RhoA gene was a functional target of miR-125a-3p. 
A

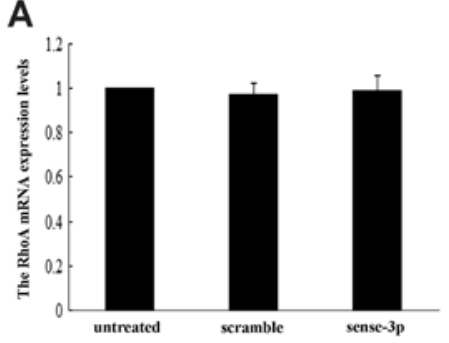

B

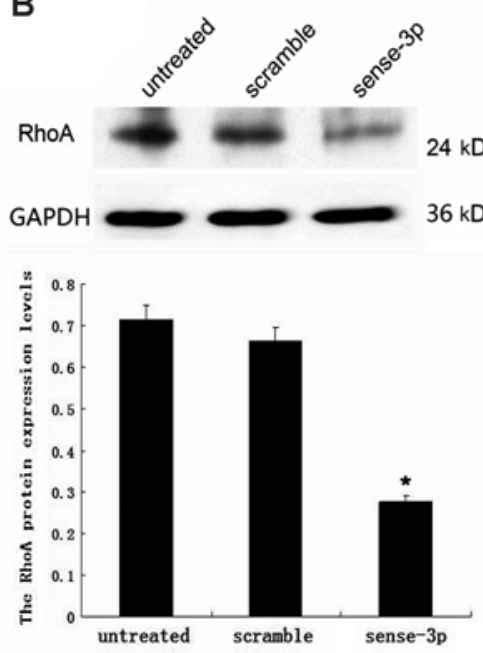

C

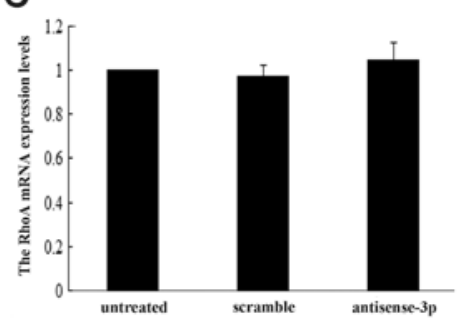

D
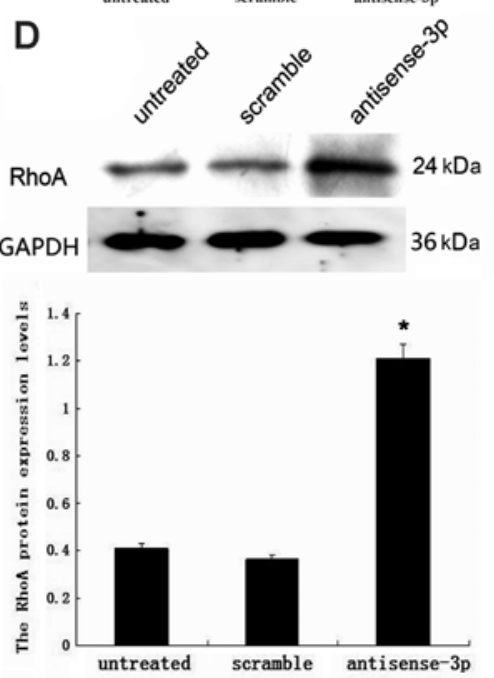

Figure 2. Effects of miR-125a-3p on RhoA expression. (A) qRT-PCR analysis showed that the expression levels of RhoA mRNA remained unchanged, when cells were transfected with sense-miR-125a-3p (sense-3p). The relative quantity of RhoA, normalized to $\beta$-actin, were compared to untreated group based on the equation $R Q=2^{-\Delta \Delta C t}$. (B) Western blot analysis showed that the expression levels of RhoA protein in the sense-miR-125a-3p transfected group was significantly lower than in the untransfected group $(\mathrm{p}<0.001)$. (C) qRT-PCR analysis indicated that the expression levels of RhoA mRNA remained unchanged, when cells were transfected with antisense-miR-125a-3p (antisense-3p). The relative quantity of RhoA, normalized to $\beta$-actin, were compared to untreated group based on the equation $R Q=2^{-\Delta A C t}$. (D) Western blot analysis showed that the expression levels of RhoA protein was higher in cells transfected with antisense-miR-125a-3 ( $<<0.001$ ).

A

3'- ccgaggguucuUGGAGUGGACa-5' miR-125a-3p 861: 5' -uggcauaaccuGUCUCACCUGg-3' RhoA

3' -ccgaggguucuUUGGAGUGGACa- $5^{\prime} \quad$ miR-125a-3p 861: 5' -uggcauaaccu ĠUCACUCGUCg- 3' RhoA 3' UTR-Mut

B

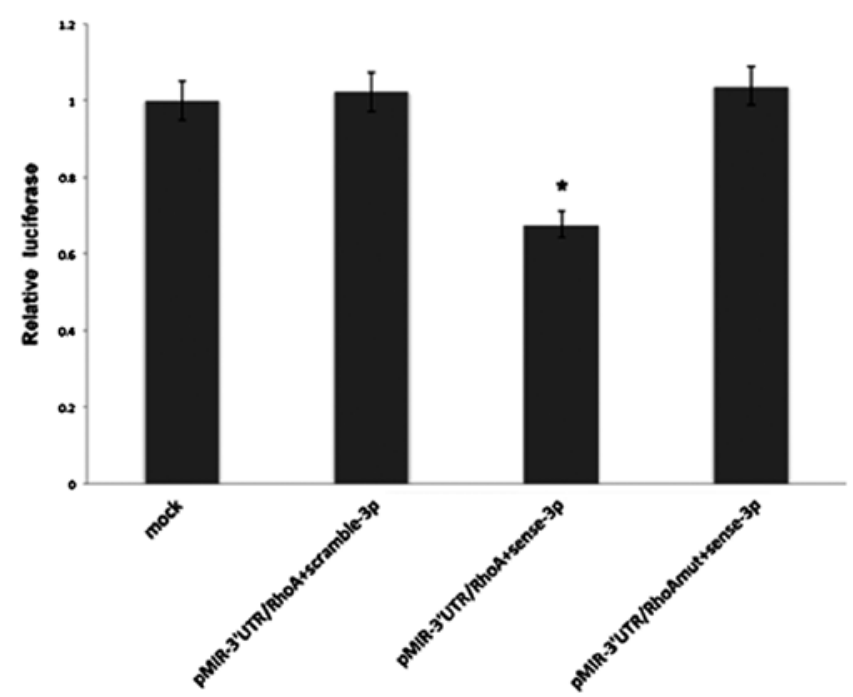

Figure 3. Interaction of miR-125a-3p and the 3'UTR of RhoA. (A) A luciferase reporter containing bioinformatics predicted interactions of miR-125a-3p and their binding sites at the 3'UTR of RhoA. A luciferase reporter containing bioinformatics predicted interactions of miR-125a-3p and their binding sites mutated at the 3'UTR of RhoA (lightgrey font). (B) miR-125a-3p inhibits the luciferase activity of a reporter containing the 3'UTR of RhoA (p<0.001) when sense-miR-125a-3p were transfected in A549. miR-125a-3p can not inhibit luciferase activity of a reporter containing a mutated sequence with the predicted target sites of the 3'UTR of the RhoA gene. 

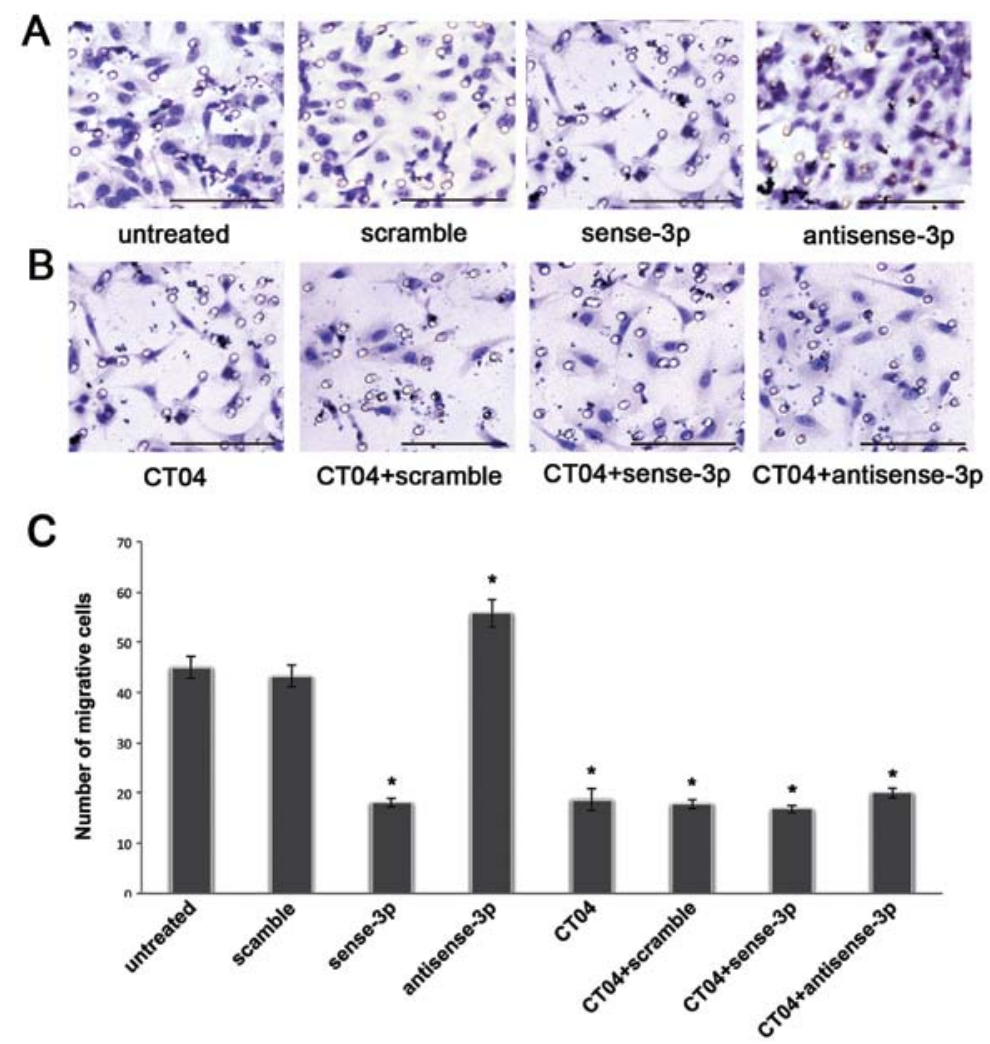

Figure 4. Effects of a Rho inhibitor on the migratory ability of A549 cells by Transwell assay. (A and C) Migration assay results show that the number of A549 cells migrating through a microporous membrane were significantly decreased after transfected sense-miR-125a-3p and increased after transfected anti-sensemiR-125a-3p. (B and C) Migration assay results show that the number of A549 cells migrating through a microporous membrane were inhibited after treatment with the Rho inhibitor, CT04, as compared with the untreated group $(\mathrm{p}<0.001)$.
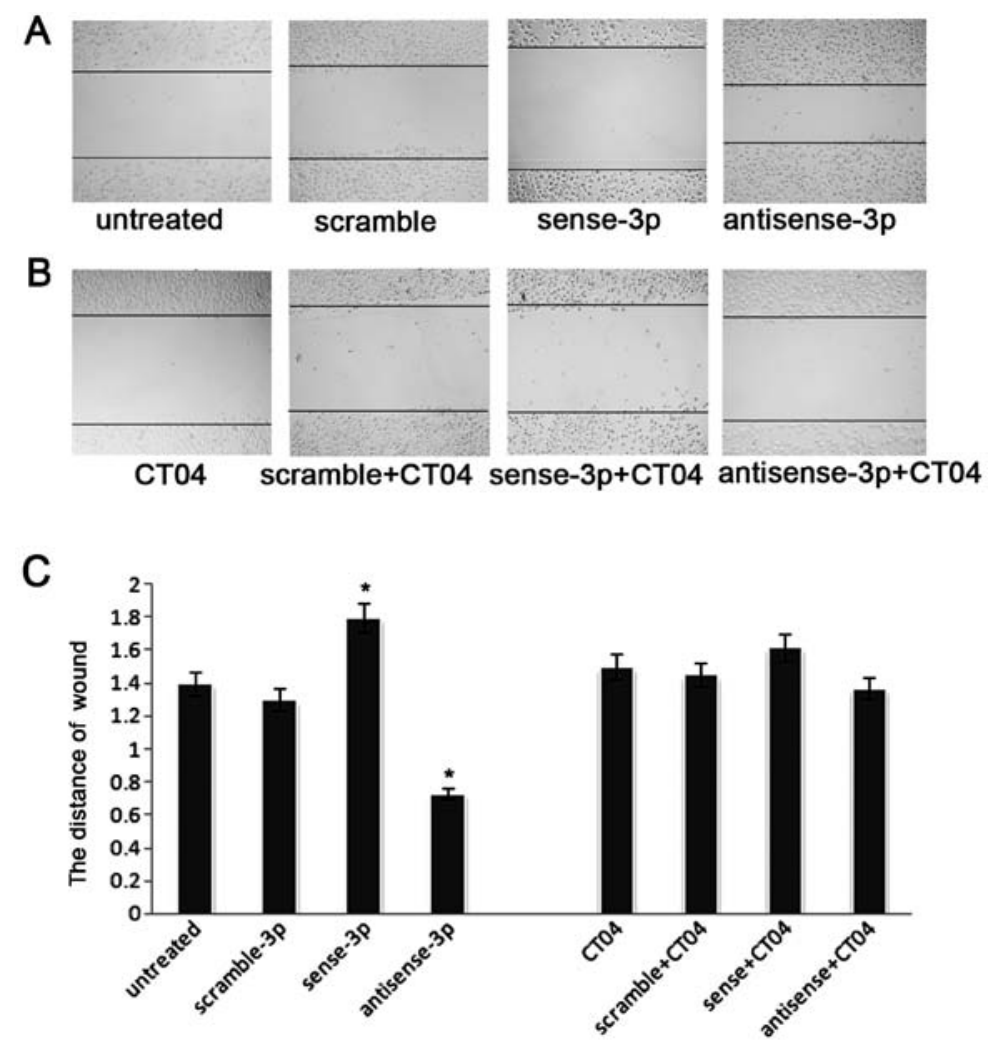

Figure 5. Effects of a Rho inhibitor on the migratory ability of A549 cells by wound healing assay. (A and C) Wound healing assay results showed that the distance of migration in A549 cells were significantly decreased after transfected sense-miR-125a-3p and increased after transfected anti-sense-miR-125a-3p. (B and C) Wound healing assay results showed that the distance of migration in A549 cells were inhibited after treatment with the Rho inhibitor, CT04, as compared with the untreated group $(\mathrm{p}<0.001)$. 
A
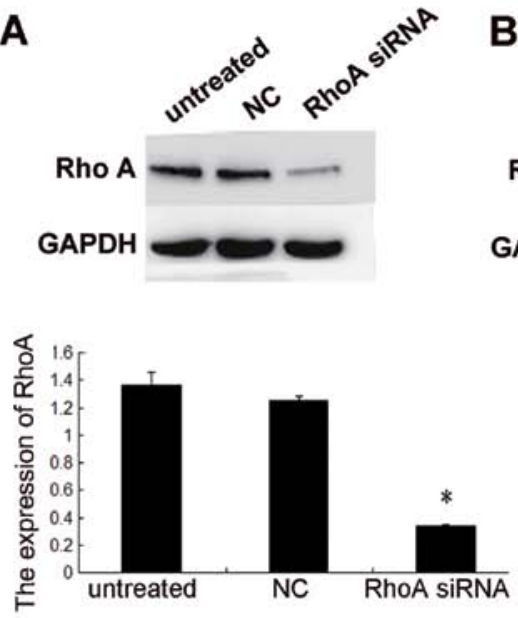

C

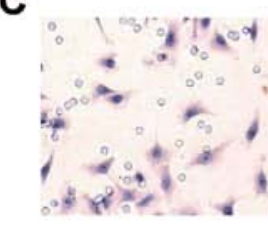

untreated

D

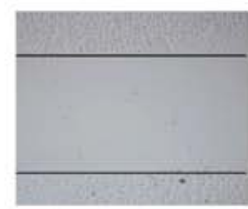

untreated

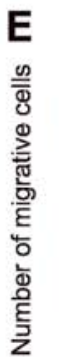

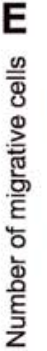

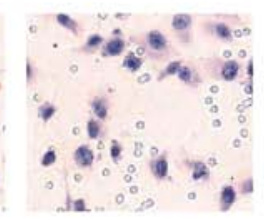

scramble

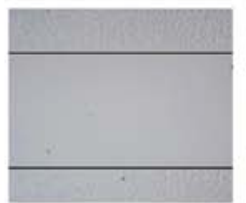

scramble
B

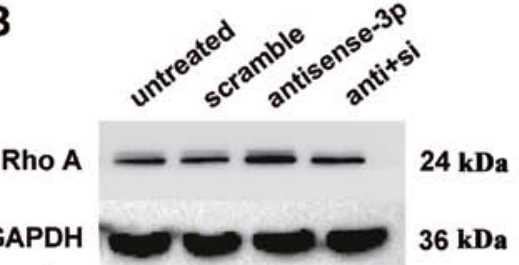

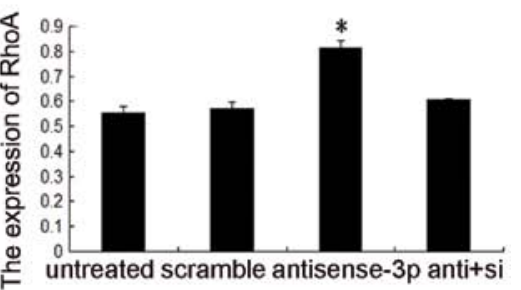



antisense-3p

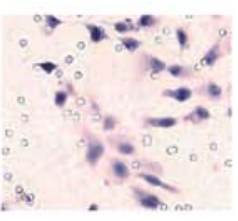

anti+si

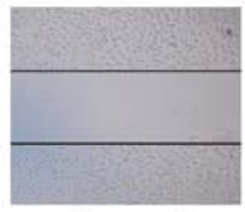

antisense-3p

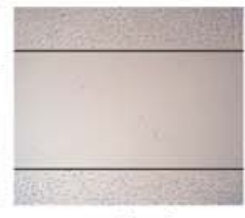

anti+si

$\mathbf{F}$



Figure 6. Effects of RhoA siRNA on the migratory ability of A549 cells. Western blot analysis shows that the expression levels of RhoA protein in the siRNA transfected group was significantly lower than in the negative control group ( $<<0.001)$. (B) The upregulation of RhoA expression mediated by antisense-miR-125a-3p was abrogated in the co-transfection with RhoA siRNA (anti+si). (C and E) Migration assay results showed that knockdown of RhoA expression abolished antisense-miR-125a-3p induced A549 cell migration. (D and F) Wound healing assay results show that knockdown of RhoA expression abolished antisense-miR125a-3p induced A549 cell migration.

MiR-125a-3p inhibits migration via the Rho-dependent pathway. Previously we found that downregulation of miR-125a-3p induced increased migration of A549 cells in transwell experiments (14). Fig. 4A shows that the number of migrating cells decreased after transfection with sense miR-125a-3p. In contrast, the number of migrating cells increased after transfection with antisense miR-125a-3p. A similar trend was seen in the wound healing assays (Fig. 5A). To further validate whether miR-125a-3p inhibits migration in a Rho-dependent manner, we blocked RhoA activity using the Rho inhibitor CT04 at a final concentration of $2.0 \mu \mathrm{g} / \mathrm{ml}$. The number of migrating cells was significantly decreased after treatment with CT04 compared with the untreated cells in a Transwell experiment without matrigel ( $<<0.001$, Fig. 4B and $C)$, and as seen in the wound healing assay $(\mathrm{p}<0.001$, Fig. $5 \mathrm{~B}$ and $\mathrm{C})$. Moreover, after treatment with CT04, the migratory ability of A549 cells transfected with the antisense-miR-125a-3p or the sense miR-125a-3p was neither decreased nor increased ( $p<0.001$, Fig. 4B and C, Fig. 5B and $\mathrm{C}$ ). These results suggest that miR-125a-3p cannot regulate RhoA when the Rho pathway is blocked.

Downregulation of RhoA by the inhibitor CT04 may directly affect the function of RhoA, independent of the concentration of protein. To rule out this effect, siRNA was used for specific downregulation of the expression of RhoA to confirm that miR-125a-3p mediated NSCLC cell migration through RhoA. The knockdown efficacy was identified using western blot 
A

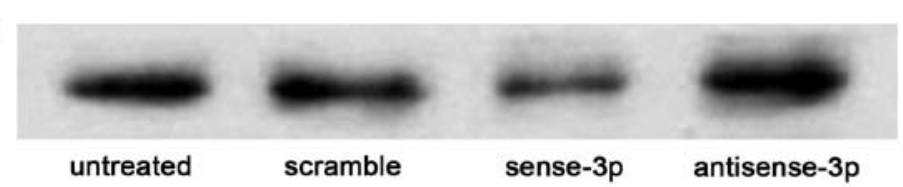

B

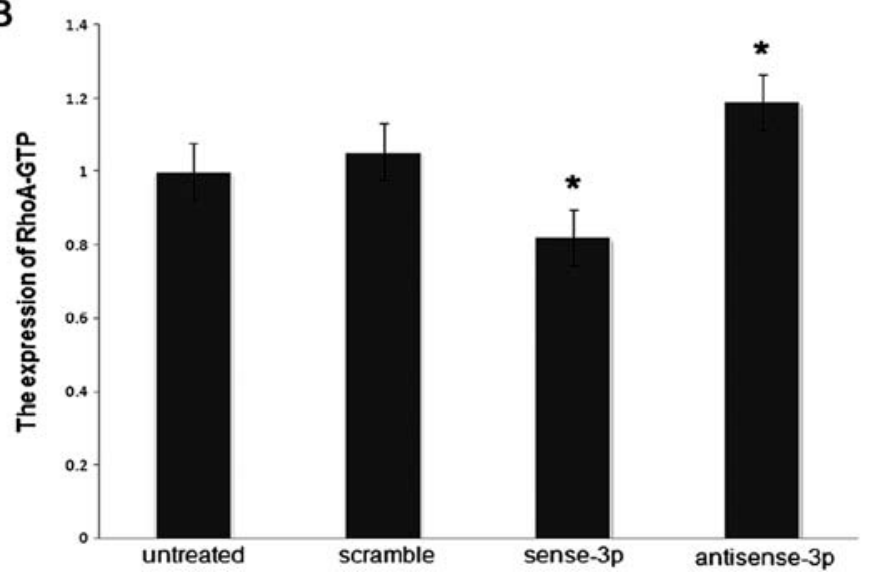

Figure 7. Effects of RhoA expression on activated RhoA (GTP-RhoA). The expression levels of GTP-RhoA were decreased when the expression levels of RhoA were declined after transfected with sense-miR-125a-3p in A549 cells; the expression levels of GTP-RhoA were increased when the expression levels of RhoA were increased after transfected with antisense-miR-125a-3p in A549 cells.

analysis (p<0.001, Fig. 6A). Knockdown of the expression of RhoA (p<0.001, Fig. 6B), abolished the effect of antisense-miR125a-3p on cell migration (Fig. 6C and D).

Effects of RhoA expression on activated RhoA (RhoA-GTP) and intracellular actomyosin. We investigated whether RhoA expression affected the migration of A549 cells via organization of the actin cytoskeleton, and also affected the levels of RhoA-GTP. The levels of RhoA-GTP were quantified using a pull-down assay after transfecting A549 cells with sense-miR-125a-3p or antisense-miR-125a-3p. The results showed that the levels of RhoA-GTP were decreased ( $p<0.001$, Fig. 7) along with decreased expression of RhoA when sense-miR-125a-3p was transfected. In addition immunofluorescence microscopy demonstrated that accumulative actin filaments were lower (Fig. 8). By contrast, the levels of RhoA-GTP increased (Fig. 7) with increase in RhoA expression when antisense-miR-125a-3p was transfected. Moreover, immunofluorescence microscopy results demonstrated that the accumulative actin filaments had grown (Fig. 8).

\section{Discussion}

MiRNAs negatively control downstream targeted genes to interfere with cell activities. Therefore, the functions of miRNAs are very important, especially with regard to their modulation of target genes. In this study, we first used bioinformatics to determine that RhoA is closely associated with cell migration and is a target gene regulated by miR-125a-3p. By manipulating miR-125a-3p expression levels in A549 NSCLC cells, we found that miR-125a-3p can post-transcriptionally regulate the expression of RhoA mRNA and repress expression of the RhoA protein. Luciferase reporter assays using reporter constructs containing the RhoA 3'UTR or specific mutated sites of RhoA 3'UTR indicated that miR-125a-3p directly targets the RhoA 3'UTR in A549 cells.

RhoA was the first identified member of the Rho family of GTPases (19). It shuttles between an inactive GDP-bound state and an active GTP-bound state and exhibits intrinsic GTPase activities, mainly involved in forming stress fibers and assembling focal adhesion complexes. Results of research using stable transfectants of active RhoA have demonstrated that the RhoAactomyosin pathway plays a pivotal role in transmigration (20). RhoA regulates the signal transduction from cell surface receptors to intracellular target molecules and is involved in a variety of biological processes, including cell morphogenesis (21), motility $(22)$, cytokinesis $(23,24)$ and tumor progression $(25,26)$. The inconsistency of the degree of RhoA transcription and levels of protein expression suggest that RhoA may be subject to post-transcriptional regulation by microRNAs. Fritz et al first detected the expression of Rho GTPases in human tumor tissues, including lung cancer. They proposed that RhoA may be overexpressed in certain tumors (27). RhoA has also been demonstrated to play an important role in tumor invasion and metastasis by its mediation of the cytoskeletal reorganization of key regulatory proteins. Results of our previous and current Transwell experiments suggest that miR-125a-3p directly targets RhoA, and may be involved in migration of NSCLC cells.

Thus, miR-125a-3p may act as an anti-oncogene by suppressing cell migration via the post-transcriptional repression of RhoA. To the best of our knowledge, this is the first study to demonstrate that miR-125a-3p impacts migration by regulating the 3'UTR of RhoA in human lung cancer A549 cells. After we blocked RhoA activity with the Rho inhibitor CT04, we found that miR-125a-3p could not regulate RhoA. In addition, the expression of RhoA was knocked down using siRNA. We found that downregulation of RhoA expression by siRNA significantly abrogated antisense-miR-125a-3p- 

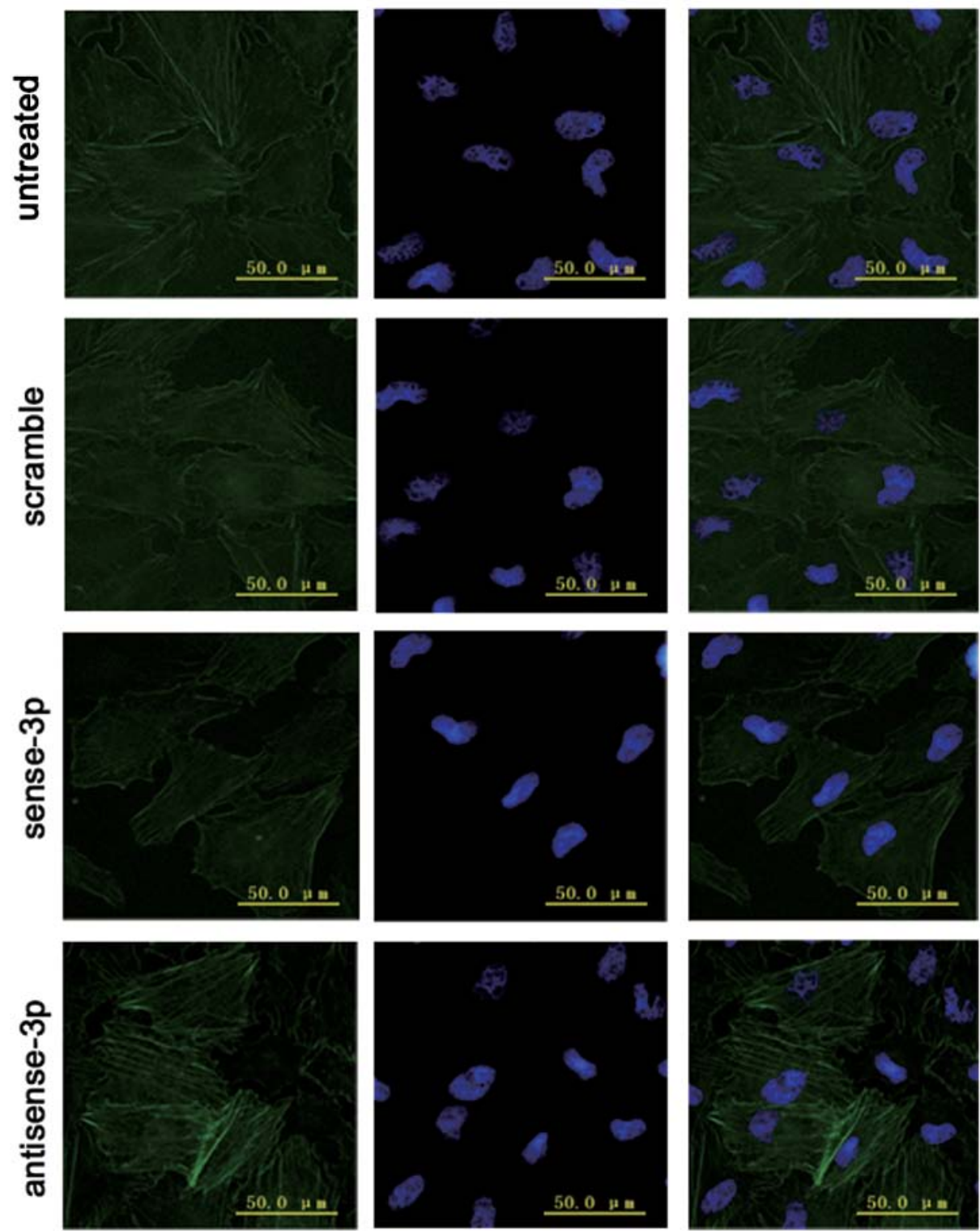

Figure 8. Effects of GTP-RhoA expression on accumulative actin filaments. As compared to the accumulative actin filaments in untransfected cells or transfected scramble cells, the results from immunofluorescence assays showed that accumulative actin filaments were more reduced with transfection of sense-miR-125a-3p. Accumulative actin filaments increased with transfection of antisense-miR-125a-3p.

induced cell migration. These results further validated the possibility that miR-125a-3p inhibited migration via a Rho-dependent pathway.

The effects of RhoA protein levels on activated RhoA have been reported. There is a report (28) that the stable RhoA transfectant of the ovarian cancer cell line SKOV3 resulted in overexpression of RhoA, which did not alter proliferative activity, but significantly increased invasiveness. The invasiveness was suppressed by the addition of a Rho inhibitor. A nude mouse model was used to show that the frequency of dissemination and the number of disseminated lesions were significantly increased in mice inoculated with RhoA transfectants compared tocontrol mice. The investigators also revealed that overexpression of RhoA mRNA and protein were associated with the spread of ovarian cancer, and believed that overexpression of RhoA had facilitated the accumulation of RhoA protein in the cell membrane and further to activate
RhoA (28). A similar conclusion was also demonstrated from work using a rat liver cell model of MM1 (29). Overexpression of RhoA had also resulted in increased levels of activated RhoA, and further enhanced tumor cell motility.

The results of our study led us to conclusions similar to these earlier reports. Upregulated expression of miR-125a-3p negatively inhibited the expression of RhoA protein because of incomplete complementation to nucleotides within the 3'UTR of the target gene RhoA. At the same time, the level of RhoA-GTP decreased in A549 cells, along with decreasing accumulative actin filaments, as shown by immunofluorescence. Downregulation of miR-125a-3p expression led to increased levels of RhoA-GTP and increased expression of RhoA protein. In addition, accumulative actin filaments increased.

We suggest that in the lung cancer cell line A549, miR$125 \mathrm{a}-3 \mathrm{p}$ directly regulates the target gene RhoA by incomplete complementation with the 3'UTR, ultimately inhibiting the 
expression levels of the RhoA protein. The level of RhoA-GTP is directly correlated with the level of RhoA protein, as well as the accumulation of actin filaments in A549 cells, which affects cell motility. MiR-125a-3p may indirectly affect the level of RhoA-GTP by direct regulation of RhoA, and thereby controls the migration of cells.

In this study, we demonstrated that miR-125a-3p posttranscriptionally regulates the 3'-UTR of RhoA mRNA and inhibits expression of RhoA protein, which indirectly decreases the levels of RhoA-GTP. Furthermore, downregulation of miR-125a-3p led to increasing accumulation of actin filaments, ultimately leading to increased migratory capacity of A549 cells. It is likely that miR-125a-3p is involved in the RhoAactomyosin pathway, which affects the migration of the lung cancer cell line A549.

\section{Acknowledgements}

We are very grateful to Dr William Kong and Dr Jin Q. Cheng for providing the plasmid containing the full-length RhoA 3'UTR used in this study. We are very grateful to Dr Baoshen Zhou from the Department of Epidemiology, School of Public Health, China Medical University for statistical analysis. We also thank Nan Liu for technical assistance, and the members of our lab for useful suggestions. This study was supported by grants from the National Natural Science Foundation of China (no. 30972967), Specialized Research Fund for the Doctoral Program of Higher Education (no. 20092104110018), Program for Liaoning Excellent Talents in University, Liaoning Provincial Natural Science Foundation (no. 20102122), and Shenyang Science and Technology Program (F10-149-9-41).

\section{References}

1. Sporn MB: The war on cancer. Lancet 347: 1377-1381, 1996.

2. Nguyen DX, Bos PD and Massague J: Metastasis: from dissemination to organ-specific colonization. Nat Rev Cancer 9: 274-284, 2009.

3. Crawford M, Brawner E, Batte K, et al: MicroRNA-126 inhibits invasion in non-small cell lung carcinoma cell lines. Biochem Biophys Res Commun 373: 607-612, 2008.

4. Xiong BH, Cheng Y, Ma L and Zhang CQ: Mir-21 regulates biological behavior through the PTEN/PI3K/AKT signaling pathway in human colorectal cancer cells. Int J Oncol 42: 219-228, 2013

5. Tavazoie SF, Alarcón C, Oskarsson T, et al: Endogenous human microRNAs that suppress breast cancer metastasis. Nature 451: 147-152, 2008

6. Nicoloso MS, Spizzo R, Shimizu M, et al: MicroRNAs - the micro steering wheel of tumour metastases. Nat Rev Cancer 9: 293-302, 2009.

7. Valastyan S, Reinhardt F, Benaich N, et al: A pleiotropically acting microRNA, miR-31, inhibits breast cancer metastasis. Cell 137: 1032-1046, 2009.

8. Bartel DP: MicroRNAs: target recognition and regulatory functions. Cell 136: 215-233, 2009.
9. Asangani IA, Rasheed SA, Nikolova DA, et al: MicroRNA-21 (miR-21) post-transcriptionally downregulates tumor suppressor Pdcd4 and stimulates invasion, intravasation and metastasis in colorectal cancer. Oncogene 27: 2128-2136, 2008.

10. He XY, Chen JX and Zhang Z: The let-7a microRNA protects from growth of lung carcinoma by suppression of $\mathrm{k}$-Ras and c-Myc in nude mice. J Cancer Res Clin Oncol 136: 1023-1028, 2010.

11. $\mathrm{Wu} \mathrm{L}$ and Belasco JG: Micro-RNA regulation of the mammalian lin-28 gene during neuronal differentiation of embryonal carcinoma cells. Mol Cell Biol 25: 9198-9208, 2005.

12. Laneve P, Di Marcotullio L, Gioia U, et al: The interplay between microRNAs and the neurotrophin receptor tropomyosin-related kinase $\mathrm{C}$ controls proliferation of human neuroblastoma cells. Proc Natl Acad Sci USA 104: 7957-7962, 2007.

13. Cowden Dahl KD, Dahl R, Kruichak JN, et al: The epidermal growth factor receptor responsive miR-125a represses mesenchymal morphology in ovarian cancer cells. Neoplasia 11: 1208-1215, 2009.

14. Jiang L, Huang Q, Zhang S, et al: Hsa-125a-3p and hsa-miR$125 \mathrm{a}-5 \mathrm{p}$ are downregulated in non-small cell lung cancer and have inverse effects on invasion and migration of lung cancer cells. BMC Cancer 10: 318, 2010.

15. Chambers AF, Groom AC and MacDonald IC: Dissemination and growth of cancer cells in metastatic sites. Nat Rev Cancer 2: 563-572, 2002.

16. Woodhouse EC, Chuaqui RF and Liotta LA: General mechanisms of metastasis. Cancer 80 (Suppl 8): 1529-1537, 1997.

17. Ho TT, Merajver SD, Lapiere CM, et al: RhoA-GDP regulates RhoB protein stability. J Biol Chem 283: 21588-21598, 2008.

18. Ming J, Liu N, Gu Y, et al: PRL-3 facilitates angiogenesis and metastasis by increasing ERK phosphorylation and up-regulating the levels and activities of Rho-A/C in lung cancer. Pathology 41: 118-126, 2009.

19. Etienne-Manneville S and Hall A: Rho GTPases in cell biology. Nature 420: 629-635, 2002

20. Yoshioka K, Matsumura F and Akedo H: Small GTP-binding protein Rho stimulates the actomyosin system, leading to invasion of tumor cells. J Biol Chem 273: 5146-5154, 1998.

21. Paterson HF, Self AJ, Garrett MD, et al: Microinjection of recombinant $\mathrm{p} 21$ rho induces rapid changes in cell morphology. J Cell Biol 111: 1001-1007, 1990.

22. Takaishi K, Kikuchi A, Kuroda S, et al: Involvement of rho p21 and its inhibitory GDP/GTP exchange protein (rho GDI) in cell motility. Mol Cell Biol 13: 72-79, 1993.

23. Kishi K, Sasaki T, Kuroda S, et al: Regulation of cytoplasmic division of Xenopus embryo by rho p21 and its inhibitory GDP/ GTP exchange protein (rho GDI). J Cell Biol 120: 1187-1195, 1993.

24. Mabuchi I, Hamaguchi Y, Fujimoto H, et al: A rho-like protein is involved in the organisation of the contractile ring in dividing sand dollar eggs. Zygote 1: 325-331, 1993.

25. Perona R, Esteve P, Jimenez B, et al: Tumorigenic activity of rho genes from Aplysia californica. Oncogene 8: 1285-1292, 1993.

26. Prendergast GC, Khosravi-Far R, Solski P, et al: Critical role of Rho in cell transformation by oncogenic Ras. Oncogene 10: 2289-2296, 1995.

27. Fritz G, Just I and Kaina B: Rho GTPases were over-expressed in human tumors. Int J Cancer 81: 682-687, 1990.

28. Horiuchi A, Kikuchi N, Osada R, et al: Overexpression of RhoA enhances peritoneal dissemination: RhoA suppression with Lovastatin may be useful for ovarian cancer. Cancer Sci 12: 2532-2539, 2008.

29. Yoshioka K, Nakamori S and Itoh K: Overexpression of small GTP-binding protein RhoA promotes invasion of tumor cells. Cancer Res 59: 2004-2010, 1999. 